\title{
Gene expression profiles of gliomas in formalin-fixed paraffin-embedded material
}

\author{
LAM Gravendeel', JJ de Rooi ${ }^{2,3}$, PHC Eilers ${ }^{3}$, MJ van den Bent ${ }^{4}$, PAE Sillevis Smitt' and PJ French ${ }^{*, 1}$ \\ 'Department of Neurology, Erasmus Medical Center, Dr Molewaterplein 50, Rotterdam 3015 GE, The Netherlands; ' Department of Bioinformatics, \\ Erasmus Medical Center, Dr Molewaterplein 50, Rotterdam 3015 GE, The Netherlands; ${ }^{3}$ Department of Biostatistics, Erasmus Medical Center, \\ Dr Molewaterplein 50, Rotterdam 3015 GE, The Netherlands; ${ }^{4}$ Department of Neuro-oncology, Daniel den Hoed Cancer Center, Groene Hilledijk 301 , \\ Rotterdam 3075 EA, The Netherlands
}

BACKGROUND: We have recently demonstrated that expression profiling is a more accurate and objective method to classify gliomas than histology. Similar to most expression profiling studies, our experiments were performed using fresh frozen (FF) glioma samples whereas most archival samples are fixed in formalin and embedded in paraffin (FFPE). Identification of the same, expression-based intrinsic subtypes in FFPE-stored samples would enable validation of the prognostic value of these subtypes on these archival samples. In this study, we have therefore determined whether the intrinsic subtypes identified using FF material can be reproduced in FFPEstored samples.

METHODS: We have performed expression profiling on 55 paired FF-FFPE glioma samples using HUI 33 plus 2.0 arrays (FF) and Exon I.0 ST arrays (FFPE). The median time in paraffin of the FFPE samples was 14.1 years (range 6.6-26.4 years).

RESULTS: In general, the correlation between FF and FFPE expression in a single sample was poor. We then selected the most variable probe sets per gene $(n=17583)$, and of these, the 5000 most variable probe sets on FFPE expression profiles. This unsupervised selection resulted in a better concordance $\left(R^{2}=0.54\right)$ between expression of FF and FFPE samples. Importantly, this probe set selection resulted in a correct assignment of $87 \%$ of FFPE samples into one of seven intrinsic subtypes identified using FF samples. Assignment to the same molecular cluster as the paired FF tissue was not correlated to time in paraffin.

CONCLUSION: We are the first to examine a large cohort of paired FF and FFPE samples. We show that expression data from FFPE material can be used to assign samples to intrinsic molecular subtypes identified using FF material. This assignment allows the use of archival material, including material derived from large-randomised clinical trials, to determine the predictive and/or prognostic value of 'intrinsic glioma subtypes' on Exon arrays. This would enable clinicians to provide patients with an objective and accurate diagnosis and prognosis, and a personalised treatment strategy.

British Journal of Cancer (2012) I 06, 538-545. doi:I0.1038/bjc.20II.547 www.bjcancer.com

Published online 20 December 2011

(c) 2012 Cancer Research UK

Keywords: glioma; expression profiling; FFPE; molecular clustering; exon array

The classification of tumour subtypes influences treatment decisions for many types of cancer. Accuracy in classifying cancer subtypes is therefore necessary to provide patients with correct diagnosis, prognosis and an optimal treatment strategy. As histological classification is often difficult in poorly differentiated tumours, this classification method urgently needs improvement. Gene expression profiling of cancer offers an accurate and objective method for classifying cancer subtypes (Sorlie et al, 2001; Valk et al, 2004). For example, in gliomas, the most common primary brain tumour in adults, gene expression profiling has identified distinct intrinsic subtypes of gliomas (Nutt et al, 2003; Freije et al, 2004; Phillips et al, 2006; Louis et al, 2007; Shirahata et al, 2007, 2009; Gravendeel et al, 2009; Li et al, 2009; Madhavan et al, 2009; Verhaak et al, 2010). We have performed unsupervised gene expression profiling in a cohort of 276 gliomas of all histological subtypes (Gravendeel et al, 2009). In this largest

*Correspondence: Dr PJ French; E-mail: p.french@erasmusmc.nl Revised 6 October 201 I; accepted 21 November 20I I; published online 20 December 2011 single-institution study conducted to date, we identified seven molecular glioma clusters. The molecular clusters were significantly better predictor of survival than histology, and were characterised by specific genetic changes. Data were validated and confirmed on six large external data sets. When validated in prospective studies these molecular clusters could contribute to clinical decision making. However, this study was conducted using RNA isolated from fresh frozen (FF) tissue. Unfortunately, FF tissue is scarce; most of the tissue archives with matched clinical outcome data are fixed in formalin and embedded in paraffin (FFPE). RNA isolated from FFPE material is often degraded and chemically modified as a result of the archiving method (Masuda et al, 1999; Farragher et al, 2008). However, new techniques have shown promising results in genome-wide expression profiling of RNA isolated from FFPE (Hoshida et al, 2008; Linton et al, 2008, 2009; Hall et al, 2011; Mittempergher et al, 2011).

Techniques used to study gene expression with FFPE material thus far have mostly been limited to single-gene analysis with RT-qPCR. Such techniques have demonstrated to be clinically relevant on a limited set of 'classifier' genes (Ma et al, 2006; Colman et al, 2010). Other multiplex assays (DASL, Quantigene, Nanostring, 
Fluidigm) have also shown promising results using distinct classifier genes (Canales et al, 2006; Geiss et al, 2008; Hoshida et al, 2008; Linton et al, 2008, 2009; Spurgeon et al, 2008; Hall et al, 2011; Mittempergher et al, 2011). Although whole-genome approaches for degraded RNA samples have improved over the last few years, the performance of current techniques to detect more subtle differences between cancer subtypes remains to be confirmed.

In this study, we therefore have performed expression analysis using a large cohort paired FF-FFPE glioma tissue using Exon 1.0 ST 'exon' arrays (Affymetrix, Santa Clara, CA, USA). Expression profiling using such a cohort has thus far not been performed. Most expression profiling studies using FF tissues have been performed on HU133-type arrays ( $\mathrm{A}, \mathrm{A}+\mathrm{B}$ or the +2.0 version), whereas best results using FFPE samples have been obtained using the Exon 1.0 ST arrays. In this study, we therefore compare the expression of FF samples on HU133 plus 2.0 arrays with FFPE samples on Exon 1.0 ST arrays. Previous studies have demonstrated good overall correlation of HU133 Plus 2.0 with Exon 1.0 ST arrays (Okoniewski et al, 2007). We show that expression data from FFPE glioma material is concordant with expression data from matched FF tissue, and can be used for molecular profiling in gliomas. Furthermore, this molecular profiling is able to identify the subtle differences between the molecular glioma subtypes.

\section{PATIENTS AND METHODS}

\section{Patient samples}

We selected 55 paired FF-FFPE samples from the Erasmus University Medical Center glioma tumour archive. The FF and the FFPE samples were taken simultaneously from the tumour as parallel biopsies. All samples were visually inspected at the time of this study for the tumour content by the neuropathologist so that samples containing at least $80 \%$ of tumour tissue were selected. The FF samples selected were used in a previous study in which seven molecular clusters were identified (Gravendeel et al, 2009). The selection contained $\sim 10$ samples from each molecular cluster. FF expression profiling results were reported previously (Gravendeel et al, 2009). The RNA from the FF tissue was extracted and hybridised in 2008. The RNA from the FFPE tissue was extracted and hybridised in 2010. Clinical and molecular data from the glioma samples included were reported previously (Gravendeel et al, 2009). The use of patient material was approved by the Institutional Review Board of the ErasmusMC, Rotterdam, the Netherlands (nr MEC 221.520/2002/262; date of approval 22 July 2003, and MEC-2005-057, date of approval 14 February 2005). For this use, patients gave written informed consent according to the Institutional and National guidelines. The fixation method of tissue in the Erasmus MC did not change over the last 25 years.

\section{RNA from FFPE extraction}

Five sections of $10 \mu \mathrm{m}$ thick were cut from each tissue block. The High Pure RNA Paraffin Kit (Roche Applied Science, Mannheim, Germany) was used to isolate the RNA from the paraffin. After isolation the RNA was purified by ethanol precipitation (Supplementary Table 1). The quantity and integrity of the RNA was measured using a Nanodrop, and an Agilent 2100 BioAnalyzer RNA 6000 Nano Assay (Agilent Technologies, Amstelveen, the Netherlands). After total RNA isolation and purification, samples were diluted to $50 \mathrm{ng} \mu \mathrm{l}^{-1}$ and stored at $-80^{\circ} \mathrm{C}$ until use.

\section{qPCR}

We randomly selected 11 samples that were assigned to two molecular clusters based on the FF expression data (Cluster 9 $(n=5)$ and Cluster $18(n=6)$; Gravendeel et al, 2009). Four genes (two upregulated, two downregulated) that discriminate between the two subtypes were examined for differential gene expression (EMP3, SLC2A10, SUSD5 and CSMD3). These genes were identified using a $t$-test in combination with fold change. ACTB, GAPDH were used as control. All reactions were performed in duplicate. Primers and conditions are described in Supplementary Table 2.

\section{Arrays}

A total of $150 \mathrm{ng}$ per sample of the extracted RNA (FFPE) was used for the Exon 1.0 ST arrays (Affymetrix). Sample labelling and array hybridisation were performed by AROS Applied Biotechnology AS (Arhus, Denmark) according to the standard Affymetrix protocols in combination with Nugen WT-Ovation technology (FFPE V2 and Exon modules; San Carlos, CA, USA; $n=55$ ). Expression arrays (HU 133 plus 2.0 (Affymetrix) using FF material was reported previously (Gravendeel et al, 2009).

Quantile normalised robust multichip average (RMA) expression levels of 22011 genes and 287329 exons were extracted from Affymetrix Exon 1.0 ST arrays using Expression Console (Affymetrix). ClusterRepro (an R package) was used to assign a sample to a defined molecular subtype (Kapp and Tibshirani, 2007).

\section{Statistics}

Differences between Kaplan-Meier survival curves were calculated by the log-rank (Mantel-Cox) test. Differences in age and RINscores of the tissue blocks were calculated using a $t$-test and a Mann-Whitney test. Significance of correlation coefficients was calculated using the $P$-value calculator for correlation coefficients (http://www.danielsoper.com/statcalc3).

\section{RESULTS}

\section{Sample characteristics}

At total of 55 FFPE samples were included in the study. These samples included 29 glioblastomas, 5 astrocytomas grade III, 5 as grade II, 4 mixed oligoastrocytoma grade III (OA II), 2 OAs grade II, 8 oligodendrogliomas grade III and 2 pilocytic astrocytoma. The median time in paraffin of the FFPE samples was 14.1 years (range 6.6-26.4 years). The median RIN score of the RNA was 2.4 (range 1.1-2.7). Sample characteristics are listed in Table 1.

\section{qPCR}

We first aimed to determine whether differences identified using expression profiling on snap frozen tissue could be found on RNA isolated from FFPE samples. For this initial test, we selected for samples that were assigned to two distinct molecular clusters based on the FF expression data (Cluster $9(n=5)$ and Cluster $18(n=6)$; Gravendeel et al, 2009). Cluster 9 shows a favourable prognosis compared with the other clusters, and is specific for loss of heterozygosity of $1 \mathrm{p}$ and $19 \mathrm{q}$, as well as a high frequency of IDH1 mutations. Cluster 18 has poor prognosis and is characterised by EGFR amplifications and CDKN2A deletions. The RT-qPCR results showed that both direction and fold change of all four genes in all samples could be recapitulated on RNA isolated from FFPE samples. The overall correlation was relatively strong $r^{2}=0.61(P<0.001)$. Correlations $\left(r^{2}\right)$ and $P$-values for individual genes EMP3, SUSD5, CSMD3 and SLC2A10 were $0.34(P=0.024)$, $0.840(P<0.001), 0.849(P<0.001)$ and $0.255(P=0.047)$, respectively. These results demonstrate that differences in gene expression are retained in RNA isolated from FFPE samples (Figure 1). 
Table I Patient and sample characteristics

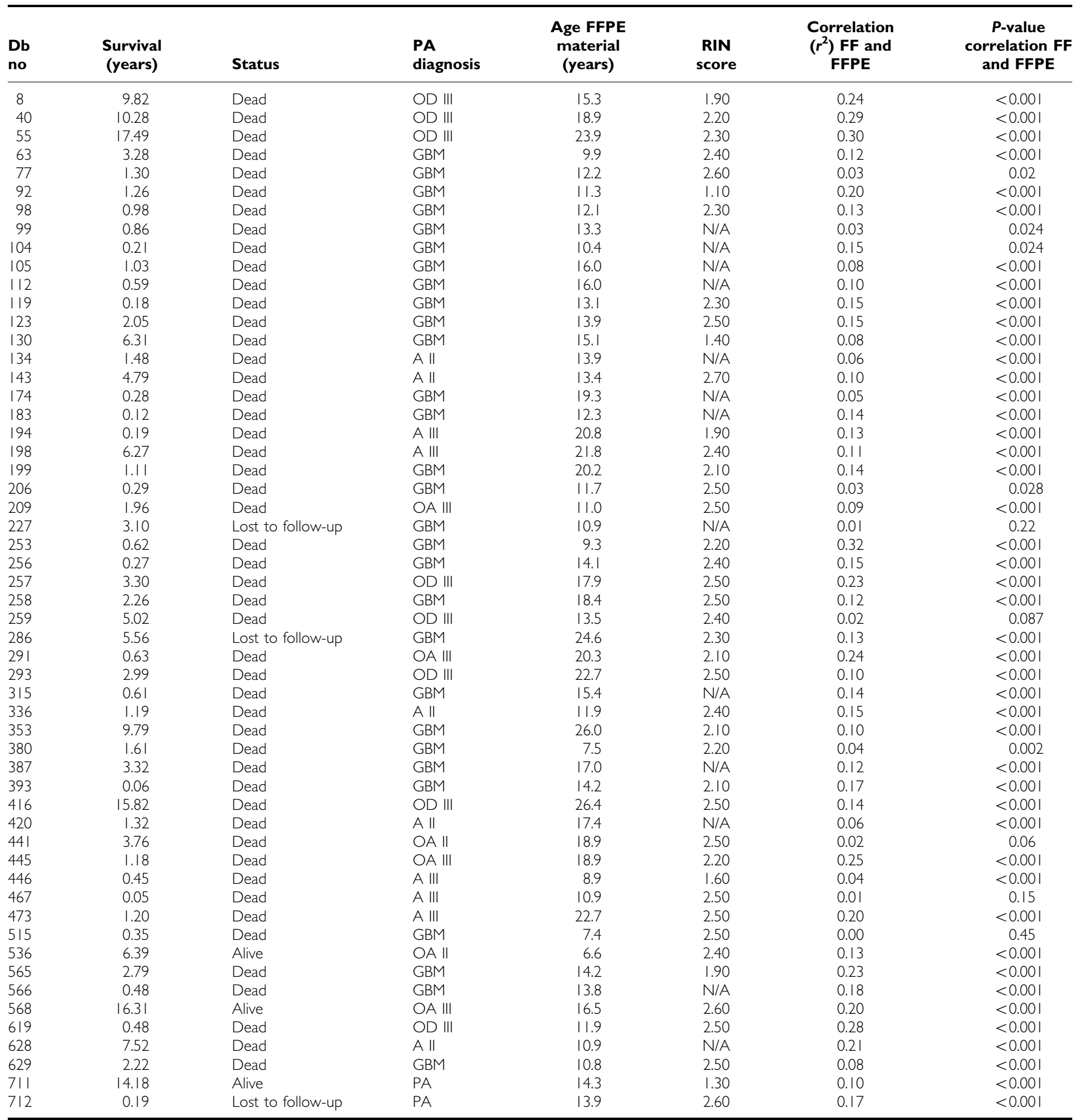

Abbreviations: A $\| l=$ astrocytoma grade $\|I ; A\|=$ astrocytoma grade $\| ; D b=$ database number; $F F=$ fresh frozen; FFPE $=$ fixed in formalin and embedded in paraffin; $\mathrm{GBM}=$ glioblastoma; OA $\|$ = oligoastrocytoma grade II; OA III = oligoastrocytoma grade III; OD I; oligodendroglioma grade II; OD II = oligodendroglioma grade III; RIN = RNA integrity number.

\section{Exon array expression data and molecular clustering analysis}

After the hybridisation of the exon 1.0 ST arrays, we compared the RMA normalised expression data of the exon arrays (FFPE) with exon 1.0 ST arrays that were analysed in earlier studies (FF tissue; French et al, 2007; Schutte et al, 2008). The exon arrays with FF tissue showed expression of more probe sets, as well as higher expression levels than the exon arrays with FFPE material (Supplementary Figure 2). On Hu133 plus 2.0 arrays (using FF samples), $9261 \pm 117(52.9 \%)$ probe sets are expressed at RMA levels $>6.5$. On exon arrays, $59618 \pm 20337(20.7 \%)$ probe sets are detected at $P<0.01$, using DABG values. It should be noted that significantly more probe sets are detected on exon arrays using FF 
A

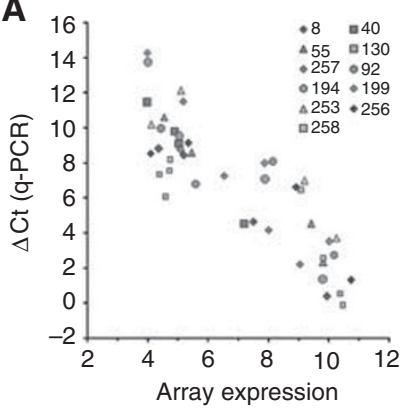

B

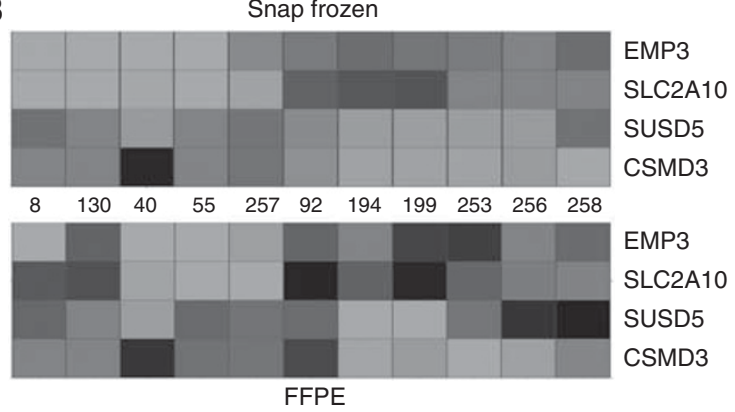

Figure I Correlation of the expression of the RNA (FFPE) and the RNA (FF) of I I matched FF-FFPE samples. The four genes (EMP3, SLC2A I0, SUSD5, CSMD3) chosen are the most discriminating genes between two very distinct molecular subtypes. (A) The correlation between the $\Delta C t$ of the qPCR results (FFPE material) and the expression data of the HUI33plus 2.0 arrays (FF tissue). A high $\Delta \mathrm{Ct}$ value is indicative for a low expression value. The correlation plot shows a good correlation between the FF expression and the FFPE expression. (B) This correlation view shows the correlation of the expression of the FF tissue (HUI 33 plus 2.0 array) and the expression of the FFPE tissue (qPCR). The green color represents low expression, red represents high expression. The molecular clusters can be identified using the RNA isolated from FFPE. The color reproduction of this figure is available at the British Journal of Cancer online.

A

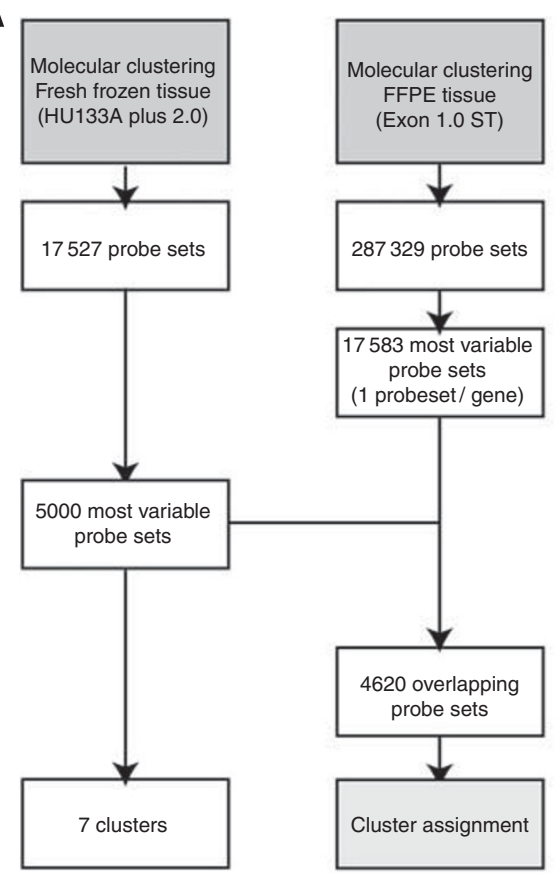

B

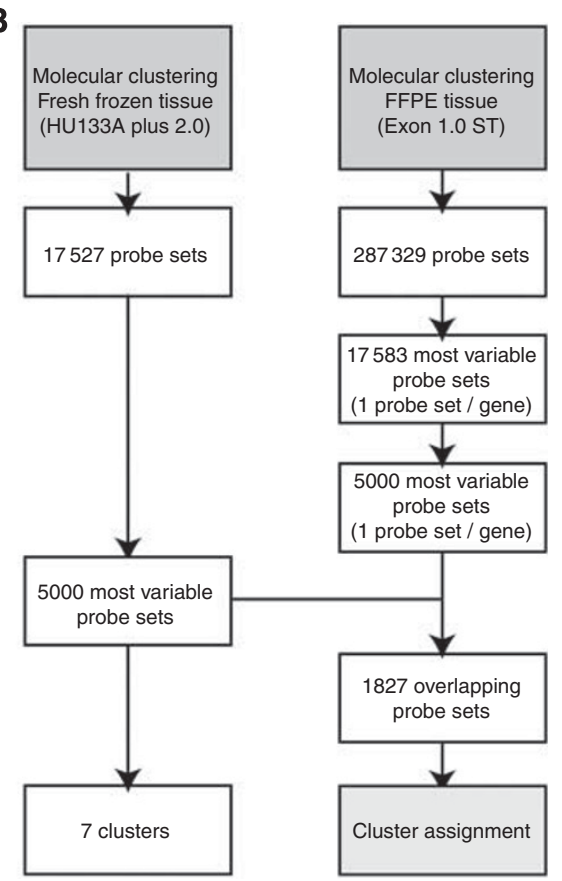

Figure 2 Flow charts of the selection of probe sets containing the most informative gene expression data. (A) We first selected the probe set with highest variance per gene $(n=17583$ probe sets; FFPE: 17583). In our previous study, we performed molecular clustering with FF tissue based on the 5000 most variable genes (FF: 5000). The overlap between the most variable probe sets in FF tissue and the most variable probe sets in FFPE tissue (FF: $5000 /$ FFPE: 17853 ) consisted of 4620 matching probe sets. On the basis of these 4620 probe sets, samples were assigned to one of the seven molecular clusters using ClusterRepro. (B) When using exon arrays, it is possible that no informative probe sets are available for a single gene, and such probe sets may be filtered out by selecting not only the most variable probe set per gene, but also, of these, the most variable 5000 probe sets (FF: 5000/FFPE: 5000). By using this filter, there are 1827 overlapping probe sets. ClusterRepro was used to assign the samples to one of the seven molecular clusters based upon these overlapping probe sets.

tissue (141 $493 \pm 12924$ (49.2\%), see also Supplementary Figure 1). In addition, the distribution of the RMA expression histograms of the FFPE glioma tissue is shifted compared with the expression histograms of exon arrays with FF tissue (Supplementary Figure 3).

\section{Correlation expression FF $v s$ FFPE}

Exon arrays contain one or more probe sets per exon for each gene (287329 core probe sets for 22011 genes), whereas only one data point per gene is generated on HU133plus2 arrays (17583 genes, when using the alternative .cdf based on entrezgene; Dai et al, 2005). We therefore first selected the probe sets on exon arrays that likely contain most of the biological information. Because genes that discriminate between molecular subtypes are by definition differentially expressed, and thus show a relatively high variance in expression, we selected the probe set with highest variance per gene ( $n=17583$ probe sets $(6.2 \%$ of all 'core' probe sets); $\log 2$ normalised data). Selecting the most variable probe set on exon arrays does not always identify those with highest correlation to expression on HU133plus2.0 arrays. However, selection based on variance approaches both data sets 
independently and avoids potential circular arguments. All further analysis was therefore done using the selection of exon array probe sets based on variance as starting data set.

In our previous study, we performed molecular clustering with FF tissue based on the 5000 most variable genes (FF: 5000). The overlap between the most variable probe sets in FF tissue and the most variable probe sets in FFPE tissue (FF: 5000/FFPE: 17 853) consisted of 4620 matching probe sets (Figure 2A).

The set of 17853 probe sets assumes that all genes have at least one informative probe set per gene. It is however possible all probe sets that belong to the same gene perform poorly. We therefore also performed a further selection of the 17583 exon array probe sets, by selecting the 5000 most variable probe sets of these 17583 ( $1.74 \%$ of the total number of 'core' probe sets, Figure 2B). Using the 5000 most variable probe sets for both FF and FFPE material showed an overlap of 1827 matching probe sets (FF: 5000/FFPE: 5000, 1827 overlapping probe sets; Figure 2B).

We first compared the gene expression data (FF) of the genes used in the qPCR analysis (EMP3, SUSD5, CSMD3 and SLC2A10) with the expression data of the FFPE material. For this analysis we used the expression data of the same 11 samples that were also used in the qPCR analysis. Figure 3 shows the correlation of the qPCR genes (EMP3, CSMD3 and SLC2A10) between the expression of the FF RNA and the FFPE RNA. These results show a weak correlation between the FF and FFPE expressions for both EMP3 and SLC2A10 $\left(r^{2}=0.32 ; P=0.028\right.$ and $\left.r^{2}=0.21 ; P=0.067\right)$, and a good correlation for CSMD3 $\left(r^{2}=0.97 ; P<0.001\right)$. There were no data available of SUSD5 as there were no probe sets of this gene on exon 1.0 ST arrays. These results highlight that exon arrays can show a concordance in gene expression compared with FF tissue, but that a selection of the biologically most informative probe sets is required.

We next compared the normalised expression data of the 5000 most variable genes as used in our previous study, with the expression of the most variable exons (FF: 5000/FFPE: 17 583, 4620 matching probe sets; Gravendeel et al, 2009). In general, the strength of correlation between FF and FFPE expression in a single sample (sample 8) was weak $\left(r^{2}=0.24, P<0.001\right)$. It should be noted that part of the between FF and FFPE sample variability is biological: The snap frozen and FFPE tissues are not taken from exactly the same location within a tumour. For this analysis we compared differential gene expression between samples of cluster 9 and 18 (separately for FF and FFPE). The correlation $\left(r^{2}\right)$ in differential gene expression between FF and FFPE was 0.38 .

We did the same analysis for the most variable 5000 probe sets (FF: 5000/FFPE: 5000, 1827 overlapping probe sets). Differential gene expression between samples of Cluster 9 and Cluster 18 then showed a relatively strong correlation between FF on HU133 plus

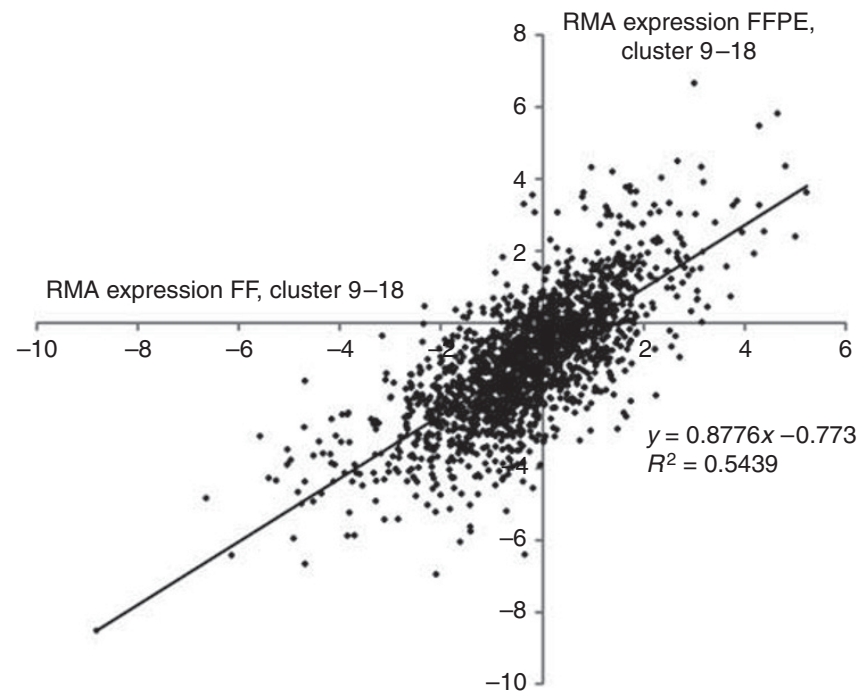

Figure 4 Differential gene expression between samples of Cluster 9 and 18. The differential gene expression between samples of two molecularly very distinct clusters (Cluster 9 and 18) showed a relatively good correlation between 18 FF samples on HUI 33 plus 2.0 and I 8 FFPE samples on HuEx I.0 ST arrays $\left(R^{2}=0.54\right)$. For this analysis we used the 5000 most variable probe sets of the exon expression data and subtracted the median expression of Cluster 18 from the median expression of Cluster 9.

2.0 and FFPE on HuEx 1.0 ST arrays $\left(R^{2}=0.54 ; P<0.001\right.$ (Figure 4). Our results demonstrate that differential gene expression between samples observed using RNA isolated from FF tissue is at least partially retained on RNA isolated from FFPE samples.

Expression data of the FF samples is performed HU133 Plus 2.0 arrays, a platform that is different from the platform used for the FFPE samples (HuEx 1.0 st arrays). We have therefore compared expression of all probes (287329) between FF and FFPE on exon arrays of eight matched samples $(8,40,130,206,257,259,275$ and 293). In general, the correlation between FF and FFPE samples on the same platform was reasonable $\left(r^{2}=0.315 \pm 0.093\right.$ range $0.210-0.450, P<0.001)$. This correlation was much better than the overall correlation (also using 287329 probe sets) between FF on HU133 Plus 2.0 arrays and FFPE on HuEx 1.0 st arrays $(0.034 \pm 0.023)$. The better correlation between FF and FFPE samples on the same platform therefore indicates that differential gene expression is better retained when using the same platform. 
Table 2 Cluster assignment of samples

\begin{tabular}{|c|c|c|c|}
\hline $\begin{array}{l}\text { Db } \\
\text { no }\end{array}$ & $\begin{array}{l}\text { Clustering } \\
\text { RNA (FF) }\end{array}$ & $\begin{array}{c}\text { Clustering } \\
\text { RNA FFPE } \\
\text { I } 7853 \text { most } \\
\text { variable exons }\end{array}$ & $\begin{array}{c}\text { Clustering } \\
\text { RNA FFPE } \\
5000 \text { most } \\
\text { variable exons }\end{array}$ \\
\hline 8 & 9 & 9 & 9 \\
\hline 40 & 9 & 9 & 9 \\
\hline 55 & 9 & 9 & 9 \\
\hline 63 & 0 & 17 & 0 \\
\hline 77 & 0 & 23 & 23 \\
\hline 92 & 18 & 18 & 18 \\
\hline 98 & 22 & 22 & 22 \\
\hline 99 & 23 & 17 & 0 \\
\hline 104 & 23 & 23 & 23 \\
\hline 105 & 23 & 23 & 23 \\
\hline 112 & 23 & 23 & 23 \\
\hline 119 & 22 & 22 & 22 \\
\hline 123 & 17 & 17 & 17 \\
\hline 130 & 9 & 9 & 9 \\
\hline 134 & 17 & 17 & 17 \\
\hline 143 & 0 & 0 & 0 \\
\hline 174 & 23 & 23 & 23 \\
\hline 183 & 23 & 23 & 23 \\
\hline 194 & 18 & 18 & 18 \\
\hline 198 & 17 & 17 & 17 \\
\hline 199 & 18 & 18 & 18 \\
\hline 206 & 22 & 0 & 0 \\
\hline 209 & 17 & 17 & 17 \\
\hline 227 & 22 & 17 & 17 \\
\hline 253 & 18 & 18 & 18 \\
\hline 256 & 18 & 18 & 18 \\
\hline 257 & 9 & 9 & 9 \\
\hline 258 & 18 & 23 & 23 \\
\hline 259 & 9 & 23 & 23 \\
\hline 286 & 17 & 17 & 17 \\
\hline 291 & 18 & 18 & 18 \\
\hline 293 & 0 & 9 & 9 \\
\hline 315 & 17 & 17 & 17 \\
\hline 336 & 22 & 9 & 0 \\
\hline 353 & 22 & 22 & 22 \\
\hline 380 & 9 & 23 & 23 \\
\hline 387 & 22 & 22 & 17 \\
\hline 393 & 23 & 23 & 23 \\
\hline 416 & 17 & 17 & 17 \\
\hline 420 & 22 & 0 & 0 \\
\hline 441 & 9 & 0 & 0 \\
\hline 445 & 18 & 18 & 18 \\
\hline 446 & 16 & 23 & 23 \\
\hline 467 & 22 & 22 & 22 \\
\hline 473 & 9 & 9 & 9 \\
\hline 515 & 23 & 23 & 23 \\
\hline 536 & 17 & 17 & 17 \\
\hline 565 & 23 & 23 & 23 \\
\hline 566 & 23 & 23 & 23 \\
\hline 568 & 17 & 17 & 17 \\
\hline 619 & 18 & 18 & 18 \\
\hline 628 & 0 & 0 & 0 \\
\hline 629 & 23 & 23 & 23 \\
\hline 711 & 16 & 16 & 0 \\
\hline 712 & 16 & 16 & 16 \\
\hline
\end{tabular}

Abbreviations: FF $=$ fresh frozen; FFPE $=$ fixed in formalin and embedded in paraffin.

\section{Cluster assignment}

Recently, we described the identification of seven molecular glioma subtypes based on gene expression profiling, which are better predictors of survival than histology (Gravendeel et al, 2009). Our final assessment to determine the suitability of RNA isolated from the FFPE samples was to confirm sample assignment to individual molecular subtypes. Clustering results are represented in Table 2. Overall, assignment to the correct cluster (e.g., assignment to the same molecular cluster as the FF tissue in the previous study) was seen in $76 \%(n=42)$ of the samples (FF: $5000 /$ FFPE: 17583). However, part of the variability between FF and FFPE samples is biological and may represent tumour heterogeneity. This heterogeneity is specifically notable for assignment to Cluster 0, as assignment to this cluster depends on the relative amount of non-neoplastic tissue present. Indeed, the overlap between FF and FFPE cluster assignment when excluding samples that are assigned to Cluster 0 is $86 \%(n=42 / 49)$. A total of 13 samples were assigned to a different molecular cluster, 7 without Cluster 0.

Similar performance in cluster assignment was observed when using the 5000 most variable exon probe sets (FF: 5000/FFPE: 5000; Figure 2B). Assignment to the identical cluster was seen in $75 \%$ ( $n=41)$ of the samples, $87 \%$ without Cluster $0(n=41 / 47)$.

Assignment to the same molecular cluster as the paired FF tissue did not have a significant correlation with the time in paraffin. The 'wrongly' assigned blocks even showed a slightly shorter median time in paraffin than the 'correctly' assigned samples (11.9 vs 14.3 years; $P=0.07)$. The average RIN score of the incorrectly assigned samples was $2.18 \pm 0.40$, and was not significantly different from the RIN scores of the correctly assigned samples $2.24 \pm 0.34, P=0.71$ ).

The high degree of overlap between FF and FFPE sample assignment (both FF: 5000/FFPE: 17583 and FF: 5000/FFPE: 5000) is reflected in a highly similar patient survival curves (Figure 5). However, FFPE survival curves also include three samples that originally were assigned to Cluster 0 .

\section{DISCUSSION}

In this study, we describe a method that allows analysis of gene expression profiling of FFPE cancer tissue using HuEx 1.0 ST arrays. Our data show that expression data of RNA isolated from FFPE and FF tissues are comparable. However, a selection on the most informative probe sets (based on highest variance for each probe set/gene and highest variance between genes) is required. RIN score and the age of the FFPE tissue blocks do not influence the gene expression results. The average RIN score and the average time in paraffin of the incorrectly assigned samples were not significantly different from the RIN scores and time in paraffin of the correctly assigned samples. The probe sets identified in this study can be used in other profiling studies that lack paired FF samples.

Differential gene expression between samples is well retained (Figure 4). This is also illustrated by the identical assignment to intrinsic molecular subtypes for both FF and FFPE glioma tissue in up to $87 \%$ of the samples (Table 2). It should be noted that FF and FFPE tissues are resected from different parts of the tumour. Therefore, tumour heterogeneity may also contribute to the differential assignment between FF and FFPE samples.

Previous studies demonstrated that FFPE samples can be used for gene expression profiling either using the Affymetrix (Exon 1.0 and HU133 plus 2.0) or using the Illumina (DASL) platforms (Hoshida et al, 2008; Linton et al, 2008, 2009; Hall et al, 2011; Mittempergher et al, 2011). However, these studies had limited sample size, lacked controlled experiments with paired FF-FFPE sample analysis or were used to differentiate between very distinct cancers. Our study is the first to use a large cohort of paired FF-FFPE glioma samples for expression profiling with exon 1.0 ST arrays. We show that degraded RNA that is up to 25 years old, is suitable to identify subtle differences between subtypes within one specific cancer.

Other genome-wide techniques are also available that can perform expression profiling on FFPE samples, including the DASL platform (Illumina). The platform chosen for this study was based on reports from literature (Linton et al, 2008, 2009), and it is beyond the scope of this manuscript to compare performance of both platforms. Although it is possible other platforms perform better on FFPE samples, our study demonstrates that sufficient 

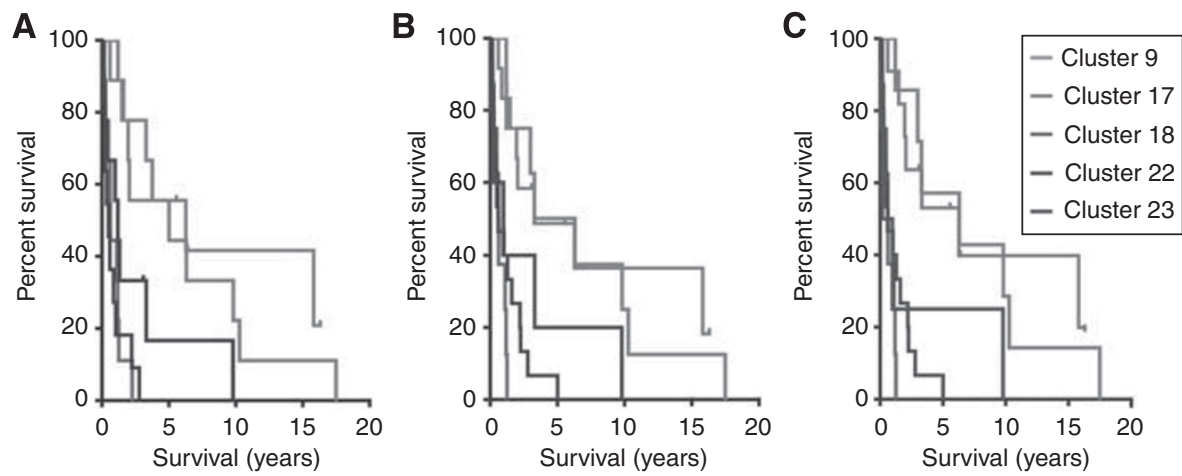

Figure 5 Kaplan-Meier survival curves of the seven molecular clusters identified in FF and FFPE material show a high resemblance. (A) shows the survival of the seven molecular clusters identified, using FF material of the 55 patients included in this study (HUI33 plus 2.0 arrays). (B) shows the survival curves of the molecular clusters to which the FFPE tissue was assigned based on the 17852 most variable probe sets from the Exon 1.0 ST arrays. (C) Shows the survival curves of the molecular clusters to which the FFPE tissue was assigned by filtering the exon data on the 5000 most variable probe sets.

information is stored in FFPE samples so that it can be used for expression profiling using exon 1.0 ST arrays. Our method allows molecular classification of archived clinical trial samples to evaluate the predictive and prognostic values of the molecular glioma clusters. Furthermore, it allows assignment of FFPE material of newly diagnosed patients to molecular clusters. Such assignment would allow clinicians to improve patients' diagnosis and would contribute to treatment decisions.

\section{ACKNOWLEDGEMENTS}

This work was supported by ZonMW project numbers 92003560 and 40-41200-98-9051, 95110051.

Supplementary Information accompanies the paper on British Journal of Cancer website (http://www.nature.com/bjc)

\section{REFERENCES}

Canales RD, Luo Y, Willey JC, Austermiller B, Barbacioru CC, Boysen C, Hunkapiller K, Jensen RV, Knight CR, Lee KY, Ma Y, Maqsodi B, Papallo A, Peters EH, Poulter K, Ruppel PL, Samaha RR, Shi L, Yang W, Zhang L, Goodsaid FM (2006) Evaluation of DNA microarray results with quantitative gene expression platforms. Nat Biotechnol 24: 1115-1122

Colman H, Zhang L, Sulman EP, McDonald JM, Shooshtari NL, Rivera A, Popoff S, Nutt CL, Louis DN, Cairncross JG, Gilbert MR, Phillips HS, Mehta MP, Chakravarti A, Pelloski CE, Bhat K, Feuerstein BG, Jenkins $\mathrm{RB}$, Aldape $\mathrm{K}$ (2010) A multigene predictor of outcome in glioblastoma. Neuro Oncol 12: 49-57

Dai M, Wang P, Boyd AD, Kostov G, Athey B, Jones EG, Bunney WE, Myers RM, Speed TP, Akil H, Watson SJ, Meng F (2005) Evolving gene/ transcript definitions significantly alter the interpretation of GeneChip data. Nucleic Acids Res 33: e175

Farragher R, Maharaj CH, Higgins BD, Crowe S, Burke P, Laffey CD, Flynn NM, Laffey JG (2008) Sevoflurane and the feto-placental vasculature: the role of nitric oxide and vasoactive eicosanoids. Anesth Analg 107: $171-177$

Freije WA, Castro-Vargas FE, Fang Z, Horvath S, Cloughesy T, Liau LM, Mischel PS, Nelson SF (2004) Gene expression profiling of gliomas strongly predicts survival. Cancer Res 64: 6503-6510

French PJ, Peeters J, Horsman S, Duijm E, Siccama I, van den Bent MJ, Luider TM, Kros JM, van der Spek P, Sillevis Smitt PA (2007) Identification of differentially regulated splice variants and novel exons in glial brain tumors using exon expression arrays. Cancer Res 67: $5635-5642$

Geiss GK, Bumgarner RE, Birditt B, Dahl T, Dowidar N, Dunaway DL, Fell HP, Ferree S, George RD, Grogan T, James JJ, Maysuria M, Mitton JD, Oliveri P, Osborn JL, Peng T, Ratcliffe AL, Webster PJ, Davidson EH, Hood L, Dimitrov K (2008) Direct multiplexed measurement of gene expression with color-coded probe pairs. Nat Biotechnol 26: 317-325

Gravendeel LA, Kouwenhoven MC, Gevaert O, de Rooi JJ, Stubbs AP, Duijm JE, Daemen A, Bleeker FE, Bralten LB, Kloosterhof NK, De Moor B, Eilers PH, van der Spek PJ, Kros JM, Sillevis Smitt PA, van den Bent MJ, French PJ (2009) Intrinsic gene expression profiles of gliomas are a better predictor of survival than histology. Cancer Res 69: 9065-9072

Hall JS, Leong HS, Armenoult LS, Newton GE, Valentine HR, Irlam JJ, Moller-Levet C, Sikand KA, Pepper SD, Miller CJ, West CM (2011)
Exon-array profiling unlocks clinically and biologically relevant gene signatures from formalin-fixed paraffin-embedded tumour samples. $\mathrm{Br} J$ Cancer 104: $971-981$

Hoshida Y, Villanueva A, Kobayashi M, Peix J, Chiang DY, Camargo A, Gupta S, Moore J, Wrobel MJ, Lerner J, Reich M, Chan JA, Glickman JN, Ikeda K, Hashimoto M, Watanabe G, Daidone MG, Roayaie S, Schwartz M, Thung S, Salvesen HB, Gabriel S, Mazzaferro V, Bruix J, Friedman SL, Kumada H, Llovet JM, Golub TR (2008) Gene expression in fixed tissues and outcome in hepatocellular carcinoma. N Engl J Med 359: 1995-2004

Kapp AV, Tibshirani R (2007) Are clusters found in one dataset present in another dataset? Biostatistics 8: 9-31

Li A, Walling J, Ahn S, Kotliarov Y, Su Q, Quezado M, Oberholtzer JC, Park J, Zenklusen JC, Fine HA (2009) Unsupervised analysis of transcriptomic profiles reveals six glioma subtypes. Cancer Res 69: $2091-2099$

Linton K, Hey Y, Dibben S, Miller C, Freemont A, Radford J, Pepper S (2009) Methods comparison for high-resolution transcriptional analysis of archival material on Affymetrix Plus 2.0 and Exon 1.0 microarrays. Biotechniques 47: 587-596

Linton KM, Hey Y, Saunders E, Jeziorska M, Denton J, Wilson CL, Swindell R, Dibben S, Miller CJ, Pepper SD, Radford JA, Freemont AJ (2008) Acquisition of biologically relevant gene expression data by Affymetrix microarray analysis of archival formalin-fixed paraffin-embedded tumours. Br J Cancer 98: 1403-1414

Louis DN, Ohgaki H, Wiestler OD, Cavenee WK (2007) WHO Classification of Tumours of the Central Nervous System, Bosman FT, Jaffe ES, Lakhani SR, Ohgaki H (eds) Lyon

Ma XJ, Patel R, Wang X, Salunga R, Murage J, Desai R, Tuggle JT, Wang W, Chu S, Stecker K, Raja R, Robin H, Moore M, Baunoch D, Sgroi D, Erlander M (2006) Molecular classification of human cancers using a 92 gene real-time quantitative polymerase chain reaction assay. Arch Pathol Lab Med 130: $465-473$

Madhavan S, Zenklusen JC, Kotliarov Y, Sahni H, Fine HA, Buetow K (2009) Rembrandt: helping personalized medicine become a reality through integrative translational research. Mol Cancer Res 7: $157-167$

Masuda N, Ohnishi T, Kawamoto S, Monden M, Okubo K (1999) Analysis of chemical modification of RNA from formalin-fixed samples and 
optimization of molecular biology applications for such samples. Nucleic Acids Res 27: 4436-4443

Mittempergher L, de Ronde JJ, Nieuwland M, Kerkhoven RM, Simon I, Rutgers EJ, Wessels LF, Van't Veer LJ (2011) Gene expression profiles from formalin fixed paraffin embedded breast cancer tissue are largely comparable to fresh frozen matched tissue. PLoS One 6: e17163

Nutt CL, Mani DR, Betensky RA, Tamayo P, Cairncross JG, Ladd C, Pohl U, Hartmann C, McLaughlin ME, Batchelor TT, Black PM, von Deimling A, Pomeroy SL, Golub TR, Louis DN (2003) Gene expression-based classification of malignant gliomas correlates better with survival than histological classification. Cancer Res 63: 1602-1607

Okoniewski MJ, Hey Y, Pepper SD, Miller CJ (2007) High correspondence between Affymetrix exon and standard expression arrays. Biotechniques 42: $181-185$

Phillips HS, Kharbanda S, Chen R, Forrest WF, Soriano RH, Wu TD, Misra A, Nigro JM, Colman H, Soroceanu L, Williams PM, Modrusan Z, Feuerstein BG, Aldape K (2006) Molecular subclasses of high-grade glioma predict prognosis, delineate a pattern of disease progression, and resemble stages in neurogenesis. Cancer Cell 9: 157-173

Schutte M, Elstrodt F, Bralten LB, Nagel JH, Duijm E, Hollestelle A, Vuerhard MJ, Wasielewski M, Peeters JK, van der Spek P, Sillevis Smitt PA, French PJ (2008) Exon expression arrays as a tool to identify new cancer genes. PLoS One 3: e3007

Shirahata M, Iwao-Koizumi K, Saito S, Ueno N, Oda M, Hashimoto N, Takahashi JA, Kato K (2007) Gene expression-based molecular diagnostic system for malignant gliomas is superior to histological diagnosis. Clin Cancer Res 13: 7341-7356

Shirahata M, Oba S, Iwao-Koizumi K, Saito S, Ueno N, Oda M, Hashimoto N, Ishii S, Takahashi JA, Kato K (2009) Using gene expression profiling to identify a prognostic molecular spectrum in gliomas. Cancer Sci 100: $165-172$

Sorlie T, Perou CM, Tibshirani R, Aas T, Geisler S, Johnsen H, Hastie T, Eisen MB, van de Rijn M, Jeffrey SS, Thorsen T, Quist H, Matese JC, Brown PO, Botstein D, Eystein Lonning P, Borresen-Dale AL (2001) Gene expression patterns of breast carcinomas distinguish tumor subclasses with clinical implications. Proc Natl Acad Sci USA 98: 10869-10874

Spurgeon SL, Jones RC, Ramakrishnan R (2008) High throughput gene expression measurement with real time PCR in a microfluidic dynamic array. PLoS One 3: e1662

Valk PJ, Verhaak RG, Beijen MA, Erpelinck CA, Barjesteh van Waalwijk van Doorn-Khosrovani S, Boer JM, Beverloo HB, Moorhouse MJ, van der Spek PJ, Lowenberg B, Delwel R (2004) Prognostically useful gene-expression profiles in acute myeloid leukemia. $N$ Engl J Med 350: 1617-1628

Verhaak RG, Hoadley KA, Purdom E, Wang V, Qi Y, Wilkerson MD, Miller CR, Ding L, Golub T, Mesirov JP, Alexe G, Lawrence M, O'Kelly M, Tamayo P, Weir BA, Gabriel S, Winckler W, Gupta S, Jakkula L, Feiler HS, Hodgson JG, James CD, Sarkaria JN, Brennan C, Kahn A, Spellman PT, Wilson RK, Speed TP, Gray JW, Meyerson M, Getz G, Perou CM, Hayes DN, Cancer Genome Atlas Research N (2010) Integrated genomic analysis identifies clinically relevant subtypes of glioblastoma characterized by abnormalities in PDGFRA, IDH1, EGFR, and NF1. Cancer Cell 17: 98 - 110

This work is published under the standard license to publish agreement. After 12 months the work will become freely available and the license terms will switch to a Creative Commons Attribution-NonCommercial-Share Alike 3.0 Unported License. 